\title{
Thermomechanical Analysis of Large Deflection of Shear Deformable FG-CNT Reinforced Composite Beams Using Perturbation Technique
}

\author{
Hadi Babaei ${ }^{1}$, Yaser Kiani ${ }^{2}$, and M.R. Eslami ${ }^{3}$ \\ ${ }^{1}$ Islamic Azad University South Tehran Branch \\ ${ }^{2}$ Amirkabir university of Technology \\ ${ }^{3}$ Amirkabir University of Technology
}

September 1, 2020

\begin{abstract}
Present investigation deals with the geometrically non-linear bending response of functionally graded carbon nanotube reinforced composite (FG-CNTRC) beams. It is assumed that beam is resting on a three parameter elastic foundation . Uniform thermal field is also included into the formulation and properties are considered as temperature dependent. Distribution of CNTs in the beam may be uniform or functionally graded according to linear distribution of volume fraction of CNTs across the thickness. The equivalent properties of the composite media are obtained with the aid of the refined rule of mixtures approach which is calibrated with the available data based on the molecular dynamics simulations. The governing equations of the beam are obtained based on first order, third order and sinusoidal theories. To capture the large deformations, the von $\mathrm{K} \backslash$ 'arm $\backslash$ 'an type of kinematic assumptions are included. Two types of boundary conditions are included which are immovable pinned and immovable clamped. The established equations are reformulated and transferred into dimensionless presentation. After that, using the powerful two step perturbation technique, closed form expressions are provided to trace the non-linear load-deflection paths of the beam. Results of this study are compared with the available data in the open literature. After that novel results are provided to discuss the effects of boundary conditions, temperature change, foundation stiffness, slenderness ratio, CNT volume fraction and distribution profiles. It is shown that graded pattern of CNTs and volume fraction of CNT are two important factors on the geometrically nonlinear response of the CNTRC beam.
\end{abstract}

\section{Hosted file}

Babaei 13.pdf available at https://authorea.com/users/355321/articles/478618thermomechanical-analysis-of-large-deflection-of-shear-deformable-fg-cnt-reinforcedcomposite-beams-using-perturbation-technique 
figures/Fig-1/Fig-1-eps-converted-to.pdf 
figures/Fig-2R/Fig-2R-eps-converted-to.pdf 
figures/Fig-2S/Fig-2S-eps-converted-to.pdf 
figures/Fig-2T/Fig-2T-eps-converted-to.pdf 
figures/Fig-30/Fig-30-eps-converted-to.pdf 
figures/Fig-3U/Fig-3U-eps-converted-to.pdf 
figures/Fig-3X/Fig-3X-eps-converted-to.pdf 
figures/Fig-4C/Fig-4C-eps-converted-to.pdf 
figures/Fig-4P/Fig-4P-eps-converted-to.pdf 
figures/Fig-5C/Fig-5C-eps-converted-to.pdf 
figures/Fig-5P/Fig-5P-eps-converted-to.pdf 
figures/Fig-6C/Fig-6C-eps-converted-to.pdf 
figures/Fig-6P/Fig-6P-eps-converted-to.pdf 
figures/Fig-7PC/Fig-7PC-eps-converted-to.pdf 
figures/Fig-8PC/Fig-8PC-eps-converted-to.pdf 
figures/Fig-9PC/Fig-9PC-eps-converted-to.pdf 